Research in Astron. Astrophys. Vol.0 (200x) No.0, 000-000

http://www.raa-journal.org http://www.iop.org/journals/raa

Research in

(LTEX: ms-raa-2021-0005.tex; printed on May 24, 2021; 0:37)

Key words: cosmology: observations - dark ages, reionization - radio lines: general - methods: observational

\title{
External Calibrator in Global Signal Experiment for Detection of the Epoch of Reionization
}

Yan Huang ${ }^{1}$, Xiang-Ping $\mathrm{Wu}^{1,2}$, Quan $\mathrm{Guo}^{2}$, Qian Zheng ${ }^{2}$, Biying $\mathrm{Li}^{1}$, Huanyuan Shan ${ }^{2}$, Kejia Lee ${ }^{1,3}$ and Haiguang $\mathrm{Xu}^{4}$

1 National Astronomical Observatories, Chinese Academy of Sciences, 20A Datun Road, Beijing 100101, China;

2 Shanghai Astronomical Observatory, Chinese Academy of Sciences, 80 Nandan Road, Shanghai 200030, China

3 Kavli Institute for Astronomy and Astrophysics, Peking University, 5 Yiheyuan Road, Beijing 100871, China

4 School of Physics and Astronomy, Shanghai Jiao Tong University, 800 Dongchuan Road, Shanghai 200240, China

\begin{abstract}
We present a conceptual design study of external calibrators in the $21 \mathrm{~cm}$ experiment towards detecting the globally averaged radiation of the epoch of reionization (EoR). Employment of external calibrator instead of internal calibrator commonly used in current EoR experiments allows to remove instrumental effects such as beam pattern, receiver gain and instability of the system if the conventional three-position switch measurements are implemented in a short time interval. Furthermore, in the new design the antenna system is placed in an underground anechoic chamber with an open/closing ceiling to maximally reduce the environmental effect such as RFI and ground radiation/reflection. It appears that three of the four external calibrators proposed in this paper, including two indoor artificial transmitters and one outdoor celestial radiation (the Galactic polarization), fail to meet our purpose. Diurnal motion of the Galactic diffuse emission turns to be the most possible source as an external calibrator, for which we have discussed the observational strategy and the algorithm of extracting the EoR signal.
\end{abstract}

\section{INTRODUCTION}

Observational campaign for detection of the redshifted $21 \mathrm{~cm}$ line of neutral hydrogen from the dark ages, cosmic dawn (CD) and epoch of reionization (EoR) has entered into a golden era, with the recent detection of the prominent absorption feature around $70 \mathrm{MHz}$ by EDGES experiment over the HighBand spectrum (Bowman et al.2018). Both the large depth and flat-bottomed shape of this signature are incompatible with the predictions of standard cosmological model of CD and EoR. This has triggered many discussions and speculations including exotic models of dark matter interaction (e.g. Barkana 2018, Fialkov et al. 2018) and an excess radiation background above the cosmic microwave radiation (e.g. Feng \& Holder 2018, Ewall-Wice et al.2018); Fialkov \& Barkana 2019). Further observations will absolutely be needed to confirm this finding.

All the ongoing experiments towards the measurement of the globally averaged radiation of EoR (hereafter EoR experiment) such as EDGES (Bowman \& Rogers 2010, Bowman et al. 2008), 
BIGHORNS (Sokolowski et al. 2015), SCI-HI (Voytek et al.2014), LEDA (Price et al. 2018), SARAS (Patra et al. 2013), and $\mathrm{PRI}^{Z} \mathrm{M}$ (Philip et al. 2019) are all based on the dipole-type antennas and their variants. Indeed, the choice of dipole-type antennas reduces the complexity of antenna system, leaving the major tasks to be calibration of the whole receive system and removal of extremely bright foreground, the two key challenges in the EoR experiment. There have also been proposals for detection of global CD/EoR signatures with short-spacing interferometers if some dedicated configurations are employed (Presley et al. 2015, Singh et al. 2015). Otherwise, lunar occultation may offer another opportunity for interferometers to achieve the goal (Mckinley et al.2018).

Calibration in current EoR experiments is essentially accomplished by the conventional threeposition switch measurements by connecting receiver to each of the following three inputs: the ambient load, an internal noise source as calibrator, and the antenna, a process called internal calibration which is fully and easily controlled (see Singh et al 2018 for a summary). This allows the sky temperature to be observationally determined provided that the accurate knowledge of antenna response is a priori known and the whole system remains stable throughout the measurements. While receiving system in each of the EoR experiments is mounted on a metal ground plane to reduce the ground radiation and reflection (but see Bradley et al. 2019), the antenna is actually exposed to environment noise despite that site is usually chosen to be remote and radio quiet.

External calibration in the EoR experiment seeks for a spectrally smooth and broadband signal external to the receiving system that has the same radiation path and sky coverage as those of the background cosmic signal. Here we have added the sky coverage as additional constraint to distinguish the traditional individual standard sources as calibrators such as Cas A. This may allow us to avoid the beam correction in the EoR experiments, helping keep the whole system completely free of systematics. Unfortunately, none of the ongoing EoR experiments has actually used the external calibration. EDGES has once tested the diurnal motion of the Galactic emission as an external calibrator but found that it is very sensitive to the beam correction (EDGES MEMO \#21511). A drone-mounted calibrator was employed for HERA, which indeed reached the desired precision but there are some systematic errors and uncertainties that need to overcome (Jacobs et al.2016).

In this paper we explore a novel conceptual design of the external calibrator for the $21 \mathrm{~cm}$ EoR experiment, based on an underground anechoic chamber at low frequency to create a radio-quiet space for calibrating the system and meanwhile isolating the environment and ground interference. Both indoor calibrators using artificial transmitters and outdoor calibrators relying on celestial sources will be considered. We wish to design a novel EoR experiment system in terms of both theoretical constraints and engineering feasibility.

\section{INDOOR CALIBRATION: A DEEP ANECHOIC CHAMBER}

We begin with an underground anechoic chamber at low frequency to demonstrate our conceptual design. A schematic of the anechoic chamber is shown in Fig.1, which is a circular shaft with an opening/closing ceiling, designed to absorb ambient background electromagnetic radiation. The walls, ceiling and floor of the anechoic chamber are treated with wedge-shaped absorbers and further shielded by metal mesh to prevent radiation from ground. A linearly-polarized dipole antenna, optimized to operate at frequencies between $50 \mathrm{MHz}$ and $200 \mathrm{MHz}$, is positioned at the center of the floor and connected via coaxial cable to a receiver with electromagnetic shield outside the anechoic chamber. The whole experiment is implemented by following three steps:

Firstly, we conduct a noise radiation measurement by closing the ceiling to create a radio-quiet space inside the anechoic chamber, which is completely free of electromagnetic radiation and reflection. The readout of the receiver in this case gives the system noise temperature at time $t_{1}$

$$
T\left(t_{1}\right)=T_{\text {sys }}\left(t_{1}\right) \text {. }
$$

Secondly, we suspend a linearly polarized cross dipole on the ceiling, which serves as a radiometric calibration source for the receiver dipole element on the floor. This calibrator system including signal

\footnotetext{
1 http://www.haystack.mit.edu/ast/arrays/Edges/EDGES-memos/
} 

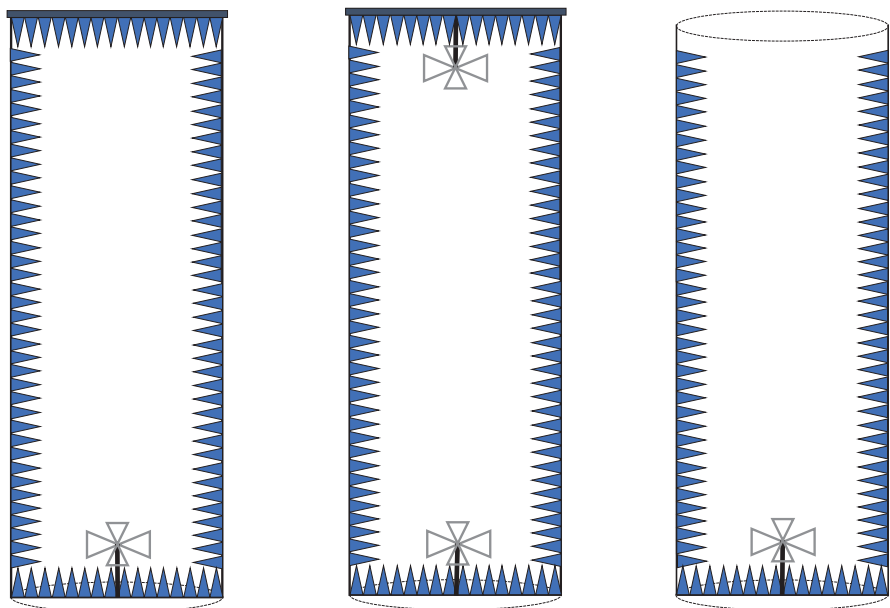

Fig. 1: A schematic of three-position switch measurements in a deep anechoic chamber

generator, transmission cable and dipole antanna should be tested and calibrated in lab before shipping. Except the dipole on the ceiling, the whole system is placed outside the anechoic chamber and electromagnetically shielded. If the vertical shaft is depth enough to meet the far-field condition, the incident radiation on the receiver antenna can be treated as a plane wave. When the calibrator is switched on, the output of the receiver can be described by a beam averaged surface brightness temperature at the frequency $\nu$ and time $t_{2}$

$$
T\left(\nu, t_{2}\right)=T_{\text {sys }}\left(t_{2}\right)+G\left(\nu, t_{2}\right)\left(1-\left|\Gamma\left(\nu, t_{2}\right)\right|^{2}\right) \frac{\int_{\Omega_{s}} A(\theta, \phi, \nu) T_{\text {cal }}\left(\theta, \phi, \nu, t_{2}\right) d \Omega}{\int A(\theta, \phi, \nu) d \Omega},
$$

where $G$ is the gain of the receiver, $\Gamma$ is the reflection coefficient accounting for the impedance mismatch between the receiver antenna and the receiver itself (Rogers \& Bowman 2012; Price et al. 2018), $A$ is the receiver antenna beam pattern, and $T_{\text {cal }}$ represents the surface brightness temperature of the calibrator. The integration in the numerator is performed over the solid angle $\Omega_{s}$ subtended by the shaft ceiling seen at the receiver antenna, while the integration in the denominator is performed over the main beam of the receiver antennas. Now we introduce the average temperature of the calibrator $\bar{T}_{\text {cal }}$ by taking the $T_{\text {cal }}$ term out of the integral, yielding

$$
T\left(\nu, t_{2}\right)=T_{\text {sys }}\left(t_{2}\right)+G\left(\nu, t_{2}\right)\left(1-\left|\Gamma\left(\nu, t_{2}\right)\right|^{2}\right) \bar{T}_{\text {cal }}\left(\nu, t_{2}\right) \frac{\int_{\Omega_{s}} A(\theta, \phi, \nu) d \Omega}{\int A(\theta, \phi, \nu) d \Omega} .
$$

Thirdly, we open the cover ceiling to allow the sky radiation to enter into the anechoic chamber. The beam averaged surface brightness temperature $T_{\text {sky }}$ now reads

$$
T\left(\nu, t_{3}\right)=T_{\mathrm{sys}}\left(t_{3}\right)+G\left(\nu, t_{3}\right)\left(1-\left|\Gamma\left(\nu, t_{3}\right)\right|^{2}\right) \frac{\int_{\Omega_{s}} A(\theta, \phi, \nu) T_{\mathrm{sky}}\left(\theta, \phi, \nu, t_{3}\right) d \Omega}{\int A(\theta, \phi, \nu) d \Omega} .
$$

Similarly, we can use the average sky temperature $\bar{T}_{\text {sky }}$ to replace $T_{\text {sky }}\left(\theta, \phi, \nu, t_{3}\right)$ such that

$$
T\left(\nu, t_{3}\right)=T_{\text {sys }}\left(t_{3}\right)+G\left(\nu, t_{3}\right)\left(1-\left|\Gamma\left(\nu, t_{3}\right)\right|^{2}\right) \bar{T}_{\text {sky }}\left(\nu, t_{3}\right) \frac{\int_{\Omega_{s}} A(\theta, \phi, \nu) d \Omega}{\int A(\theta, \phi, \nu) d \Omega} .
$$


Because the sky radiation can evidently be treated as plane wave, the above integral is performed over the same solid angle as the one in Eq.(3).

We now work with the following ratio

$$
\frac{T\left(\nu, t_{3}\right)-T_{\mathrm{sys}}\left(t_{3}\right)}{T\left(\nu, t_{2}\right)-T_{\mathrm{sys}}\left(t_{2}\right)}=\frac{G\left(\nu, t_{3}\right)\left(1-\left|\Gamma\left(\nu, t_{3}\right)\right|^{2}\right) \bar{T}_{\mathrm{sky}}\left(\nu, t_{3}\right)}{G\left(\nu, t_{2}\right)\left(1-\left|\Gamma\left(\nu, t_{2}\right)\right|^{2}\right) \bar{T}_{\mathrm{cal}}\left(\nu, t_{2}\right)}
$$

If the three-position switch measurements are implemented within a short time so that the receiver system characterized by the receiver temperature $T_{\text {sys }}(t)$, the gain $G(\nu, t)$ and the reflection coefficient $\Gamma(\nu, t)$ remain unchanged, we can get the average sky temperature at frequency $\nu$ and time $t$ through

$$
\bar{T}_{\text {sky }}=\frac{T_{3}-T_{\text {sys }}}{T_{2}-T_{\text {sys }}} \bar{T}_{\text {cal }} .
$$

The sky temperature can be decomposed into foreground contribution $\left(T_{\mathrm{f}}\right)$ including the Galaxy and extragalactic sources and background one $\left(T_{21 \mathrm{~cm}}\right)$ from EoR - the signal that we expect to detect in the experiment. For a featureless-spectrum foreground dominated by synchrotron radiation, it is possible to find the best-fitted foreground $\bar{T}_{\mathrm{f}}^{\text {fit }}$ from Eq.(7), following the mature algorithms developed in past two decades from the simple low-order polynomial fit (e.g. Wang et al. 2006, Pritchard \& Loeb 2010) to various sophisticated techniques such as the Markov Chain Monte Carlo approach (Harker et al.2012), the independent component analysis (Chapman et al.2012), and the Bayesian techniques (Bernardi et al. 2016). This allows us to extract the EoR signal simply from

$$
\bar{T}_{21 \mathrm{~cm}}=\bar{T}_{\mathrm{sky}}-\bar{T}_{\mathrm{f}}^{\mathrm{fit}} \text {. }
$$

Note that unlike all the ongoing and planned experiments which need precise knowledge of antenna beam pattern and its spatial and spectral variation, the above measurement of $T_{21 \mathrm{~cm}}$ is entirely independent of antenna property, and the external calibrator $T_{\text {cal }}$ turns to be the key component for the success of such experiment.

Now we study the feasibility of the above design by examining the sensitivity of the system represented by the RMS variation in surface brightness temperature

$$
\Delta T_{\mathrm{b}}=\frac{\lambda^{2} T_{\mathrm{sys}}}{A_{e} \Omega_{s} \sqrt{\Delta t \Delta \nu}}
$$

where $A_{e}$ is the effective area of the antenna, $\Delta t$ is the integration time, and $\Delta \nu$ is the bandwidth. The solid angle $\Omega_{s}$ subtended by the shaft ceiling is

$$
\Omega_{s}=2 \pi\left(1-\frac{D}{\sqrt{D^{2}+R^{2}}}\right),
$$

in which $R$ and $D$ represent the radius and depth of the shaft, respectively. If the system noise is dominated by the Milky Way and follows a power-law of $T_{\text {sys }}=60 \mathrm{~K}(\nu / 300 \mathrm{MHz})^{-2.55}$, the sensitivity can be quantitatively estimated through

$$
\Delta T_{\mathrm{b}}=4.82 \mathrm{mK} \frac{1}{f(R, D)}\left(\frac{\nu}{100 \mathrm{MHz}}\right)^{-4.55}\left(\frac{A_{e}}{\mathrm{~m}^{2}}\right)^{-1}\left(\frac{\Delta t}{\text { day }} \frac{\Delta \nu}{\mathrm{MHz}}\right)^{-1 / 2},
$$

where $f(R, D)=1-D / \sqrt{D^{2}+R^{2}}$ is the geometrical factor. For the experiment on the ground, $f(R, D=0)=1$ and $\Omega_{s}=2 \pi$. In this case one can easily achieve a sensitivity of a few $\mathrm{mK}$ at 100 $\mathrm{MHz}$ within a day. However, for our experiment inside an underground anechoic chamber, the calibrator antenna is assumed to share approximately the same patch of the sky as that of the cosmological signal. This requires that the depth $(D)$ of the shaft should be made to be much larger than its diameter $(2 R)$. Taking the more relaxed case of $D=2 \times 2 R$ as an example, we have $f(R, D=2 R) \approx 0.03$ and $\Omega_{s} \approx 3 \% \times 2 \pi$. To reach a detection limit of $\Delta T_{\mathrm{b}}=10 \mathrm{mK}$ at $\nu=100 \mathrm{MHz}$ with an effective area 


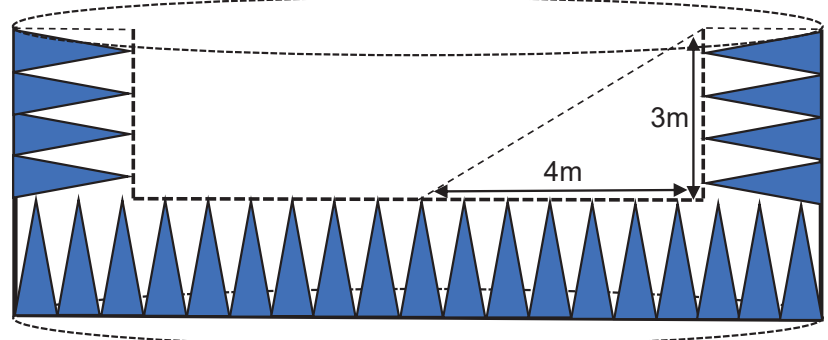

Fig. 2: An illustrative model of the shallow anechoic chamber in the open-ceiling mode.

of $A_{e}=1 \mathrm{~m}^{2}$ and a bandwidth of $\Delta \nu=1 \mathrm{MHz}$, one has to integrate the measurements at openceiling position for 261 days. Considering the fact that the real measurements is carried out in a way of three-position switch and the efficiency of operation is apparently much less than $100 \%$, we may have to implement the experiment on a time scale of up to 5-10 years in order to detect the global EoR signal. The main reason behind the long integration time is the small sky coverage $\Omega_{s}$, subtended by the shaft window, which prevents the antenna from receiving sky radiation from all directions-the key for capture of the global signature.

\section{INDOOR CALIBRATION: A SHALLOW ANECHOIC CHAMBER}

If the main conceptual design based on underground anechoic chamber in the above section is adopted, the only way to enhance the sensitivity is to increase the field angle $\Omega_{s}$ so that the antenna placed on the floor can observe a large patch of the sky. A shallow shaft design certainly meets the purpose, which however, breaks the plane-wave assumption in the case of deep shaft. In particular, a dipole antenna on the ceiling can no longer serve as a calibrator because its radiation field does not mimic the sky signals anymore. So, the adoption of a shallow shaft design means that a new calibration approach should be developed.

We begin with the geometric parameters of the shaft within the underground anechoic chamber to make sure that the sensitivity of the receiving dipole antenna on the floor can reach a detection limit of $\sim 10 \mathrm{mK}$ within a reasonable integration time, say less than a few months. While there are numerous combinations in (R, D) space to play with, here we focus on a simple choice of $R=4 \mathrm{~m}$ and $D=3 \mathrm{~m}$ to demonstrate the design. Such an illustrative model yields $f(R=4, D=3)=2 / 5$ and $\Omega_{s}=4 \pi / 5$, covering $40 \%$ of the sky above the horizon. Correspondingly, the sensitivity reads

$$
\Delta T_{\mathrm{b}}=12.1 \mathrm{mK}\left(\frac{\nu}{100 \mathrm{MHz}}\right)^{-4.55}\left(\frac{A_{e}}{\mathrm{~m}^{2}}\right)^{-1}\left(\frac{\Delta t}{\text { day }} \frac{\Delta \nu}{\mathrm{MHz}}\right)^{1 / 2} .
$$

A detection limit of $10 \mathrm{mK}$ is achievable within a few days over a broad frequency range except at low frequency end. For example, one may has to accumulate the data at the open-sky mode for about 20 days at $\nu=75 \mathrm{MHz}$. This, however, does not affect the model for the purpose of illustration.

The real challenge is the design of a different type of calibrator to replace the dipole antenna suspending on the ceiling in the case of deep shaft. Yet, the radiation field of the new calibrator should exhibit the same behaviour as that of the sky over the solid angle $\Omega_{s}$. Although there are probably other solutions to the problem, here we only explore two technical schemes to meet the requirement.

The first choice is the drone-based calibrator, which has been successfully applied in the beam calibration of radio telescope (Chang et al. 2015). In particular, this external calibration method has 
also been tested in the low frequency antennas including SKALA designed for SKA1-low (Virone et al. 2014) and low frequency array dedicated to the detection of EoR signal (e.g. Jacobs et al. 2016). While technically the method has become mature and reliable, the employment of the drone-mounted calibrator in our experiment is unfortunately impractical. An immediate reason is the violation of our indoor calibration principle: Radiation from the calibrator is contaminated by background sky when the underground anechoic chamber is exposed to ambient environment even if there is no RFI in experimental site. A more serious problem is actually the flight time to smoothly cover the field of view, $\Omega_{s}$. Although we could carefully design the flight pattern in terms of either rectangular grids or concentric tracks above the antenna to have a smooth coverage of $\Omega_{s}$, it takes rather a long time to complete the calibration process, during which there is no guarantee that the receiver system remains stable. Recall that the receiver properties characterized by $T_{\text {sys }}(t), G(\nu, t)$ and $\Gamma(\nu, t)$ are assumed unchanged in the three-position switch measurements [see Eq.(6)]. Therefore, we will not explore further the calibration with drone unless there is an independent way to measure the system temperature by adding an internal noise source.

The second approach is to place a spherical dish antenna as a new ceiling to fully cover the anechoic chamber. The spherical dish itself will act as a calibrator because it can generate an isotropic radiation field, in a similar way as the sky signal, toward the dipole antenna on the floor. Our last two-step measurements will then switch between this artificial radio 'sky' and the open sky positions, both of which subtend the same solid angle $\Omega_{s}$ seen at the dipole antenna at the floor. This requires that the spherical dish should be designed to be removed or opened conveniently and quickly in the three-position switch measurements. Taking the illustrative model of $R=4 \mathrm{~m}$ and $D=3 \mathrm{~m}$ as an example, we need a spherical dish antenna of $8 \mathrm{~m}$ in diameter with a curvature radius of $5 \mathrm{~m}$ to the focus point, which is exactly the position of the receiving dipole on the floor. So, the feed of the spherical dish should be mounted at the position of the receiving dipole, and the transmitting and reflecting signal should exhibit a spectral flatness over 50-200 MHz. Although one could use the same dipole for both transmitting and receiving purpose, the reflecting signal from the dish surface as a calibrator deviates from an isotropic radiation field. One could further explore the possibility of designing a compact feed of spherical beam symmetry over $\Omega_{s}$ in a low frequency wideband of $50-200 \mathrm{MHz}$ such as the sinuous antenna tested in the HERA experiment (e.g. Garza et al.2018). However, it is mechanically impractical to put both the feed and the receiving dipole antenna exactly at the same position.

Yet, there is a third way to generate an isotropic radiation field over $\Omega_{s}$ by combining and modifying the design concepts of the first and second method above: Instead of utilization of the feed at the focus point for the spherical dish, we can suspend a movable, dual polarized dipole antenna as a calibrator on the surface of the spherical cap. The track of the moving calibrator can be designed to maximally and smoothly cover the dish surface though the calibration is actually performed at finite and discrete grids over a certain time. A continuous calibration process is also possible if the speed of the calibrator can be precisely controlled to be constant. Another fundamental change is that the dish should be made of non-metal material to absorb radiation or eliminate reflection from the calibrator. Furthermore, the dish surface should not be covered with wedge-shaped absorbers to allow the calibrator antenna to move freely. It is easy to show that in this case our illustrating model of $R=4 \mathrm{~m}$ and $D=3 \mathrm{~m}$ satisfies the far-field requirement: the distance $(5 \mathrm{~m})$ of the calibrator to the receiving antenna is larger than twice of the square of the antenna size $(\sim 1 \mathrm{~m})$ over the shortest wavelength $(1.5 \mathrm{~m})$. While such a design seems feasible and operational, there are still two major concerns for the measurement of the global EoR signal: First, the time to complete the whole calibration process may be too long to maintain the stability of the receiver system - a key assumption to remove the receiver effect in the three-position switch. Second, the calibration can only be performed on finite grids or tracks on the disk surface, and it is never possible to do the calibration at every position of the spherical cap. Mathematical modeling should be invoked to quantity the calibration error from the discrete sampling. Therefore, the spherical dish cover with a movable calibrator antenna is still not an ideal and practical design for our purpose. 


\section{OUTDOOR CALIBRATION: DIURNAL MOTION}

We wish to keep the basic design (e.g. the illustrative model) of the underground shallow anechoic chamber in the above section to benefit from both radiation-free environment and high sensitivity, and explore the possibility of calibrating the system using outdoor sources. In this case, the anechoic chamber will work in a complete open-ceiling mode while the ceiling is nevertheless needed to create a radiation-free space to measure the system noise temperature $T_{\text {sys }}$, the so-called first step in the experiment. Man-made calibrators such as the drone-based one are difficult to meet our purpose due to the limitation of uniformity and smoothness of sky coverage and lower efficiency as discussed in Section 3. We should therefore rely on celestial sources in the low-frequency sky to fulfill the task. In particular, because the celestial calibrators should share the same sky and frequency coverage as those of the EoR signal, very bright radio sources such as the Cas A or the Moon suggested in literature (e.g. Shaver et al. 1999) cannot be chosen. The candidate is therefore the Milky Way, the brightest diffuse foreground, or extragalactic radio sources distributed isotropically in the sky.

The first step remains essentially the same as that in the indoor calibration discussed the above, and the system noise $T\left(t_{1}\right)=T_{\text {sys }}\left(t_{1}\right)$ is measured within the anechoic chamber at time $t_{1}$. The main difference arises from following steps: The anechoic chamber will receive radiation from the sky in an open-ceiling mode in the two-position switch between calibration (second step) and data acquisition (third step). Actually, we will not make a distinction between the calibration and measurement processes any more, which are carried out exactly in the same way except at different time. Denoting the possible sky variation between the latter two-position switch as $\Delta T$, we get the system response at time $t_{2}$

$T\left(\nu, t_{2}\right)=T_{\text {sys }}\left(t_{2}\right)+G\left(\nu, t_{2}\right)\left(1-\left|\Gamma\left(\nu, t_{2}\right)\right|^{2}\right) \frac{\int_{\Omega_{s}} A(\theta, \phi, \nu)\left[T_{\text {sky }}\left(\theta, \phi, \nu, t_{2}\right)-\Delta T\left(\theta, \phi, \nu, t_{2}\right) / 2\right] d \Omega}{\int A(\theta, \phi, \nu) d \Omega}$.

Introducing the average sky temperature $\bar{T}_{\text {sky }}$ and its variation $\Delta \bar{T}$ over the solid angle $\Omega_{s}$, we rewrite the above equation as

$$
T\left(\nu, t_{2}\right)=T_{\text {sys }}\left(t_{2}\right)+G\left(\nu, t_{2}\right)\left(1-\left|\Gamma\left(\nu, t_{2}\right)\right|^{2}\right)\left[\bar{T}_{\text {sky }}\left(\nu, t_{2}\right)-\Delta \bar{T}\left(\nu, t_{2}\right) / 2\right] \frac{\int_{\Omega_{s}} A(\theta, \phi, \nu) d \Omega}{\int A(\theta, \phi, \nu) d \Omega} .
$$

Similarly, the system readout at time $t_{3}$ is

$$
T\left(\nu, t_{3}\right)=T_{\text {sys }}\left(t_{3}\right)+G\left(\nu, t_{3}\right)\left(1-\left|\Gamma\left(\nu, t_{3}\right)\right|^{2}\right)\left[\bar{T}_{\text {sky }}\left(\nu, t_{3}\right)+\Delta \bar{T}\left(\nu, t_{3}\right) / 2\right] \frac{\int_{\Omega_{s}} A(\theta, \phi, \nu) d \Omega}{\int A(\theta, \phi, \nu) d \Omega} .
$$

We now work with the following ratio

$$
\frac{T\left(\nu, t_{2}\right)-T_{\mathrm{sys}}\left(t_{2}\right)}{T\left(\nu, t_{3}\right)-T_{\mathrm{sys}}\left(t_{3}\right)}=\frac{G\left(\nu, t_{2}\right)\left(1-\left|\Gamma\left(\nu, t_{2}\right)\right|^{2}\right)\left[\bar{T}_{\mathrm{sky}}\left(\nu, t_{2}\right)-\Delta \bar{T}\left(\nu, t_{2}\right) / 2\right]}{G\left(\nu, t_{3}\right)\left(1-\left|\Gamma\left(\nu, t_{3}\right)\right|^{2}\right)\left[\bar{T}_{\mathrm{sky}}\left(\nu, t_{3}\right)+\Delta \bar{T}\left(\nu, t_{3}\right) / 2\right]} .
$$

Again, if the system remains stable throughout the three-position switch measurements, the above equation reduces to

$$
\frac{T_{2}(\nu, t)-T_{\text {sys }}(t)}{T_{3}(\nu, t)-T_{\text {sys }}(t)}=\frac{\bar{T}_{\text {sky }}(\nu, t)-\Delta \bar{T}(\nu, t) / 2}{\bar{T}_{\text {sky }}(\nu, t)+\Delta \bar{T}(\nu, t) / 2} .
$$

Most importantly, it appears that the method would fail if $\Delta \bar{T}(\nu, t)=0$, indicating that it is the timevarying component that acts as the calibrator rather than the total foreground radiation. The diurnal motion of the Galactic signal has thus been suggested and tested as a calibrator in the EoR experiment to separate out the EoR signal by EDGES (Memo \#048, \#055, \#202 \& \#2152). However, this method has not been adopted by EDGES mainly due to its sensitive dependence on beam correction. SCI-HI (Voytek et al.2014) also applied the 24 hour data of the Galactic variation to calibrate the total power spectrum.

\footnotetext{
${ }^{2}$ http://www.haystack.mit.edu/ast/arrays/Edges/EDGES-memos/
} 


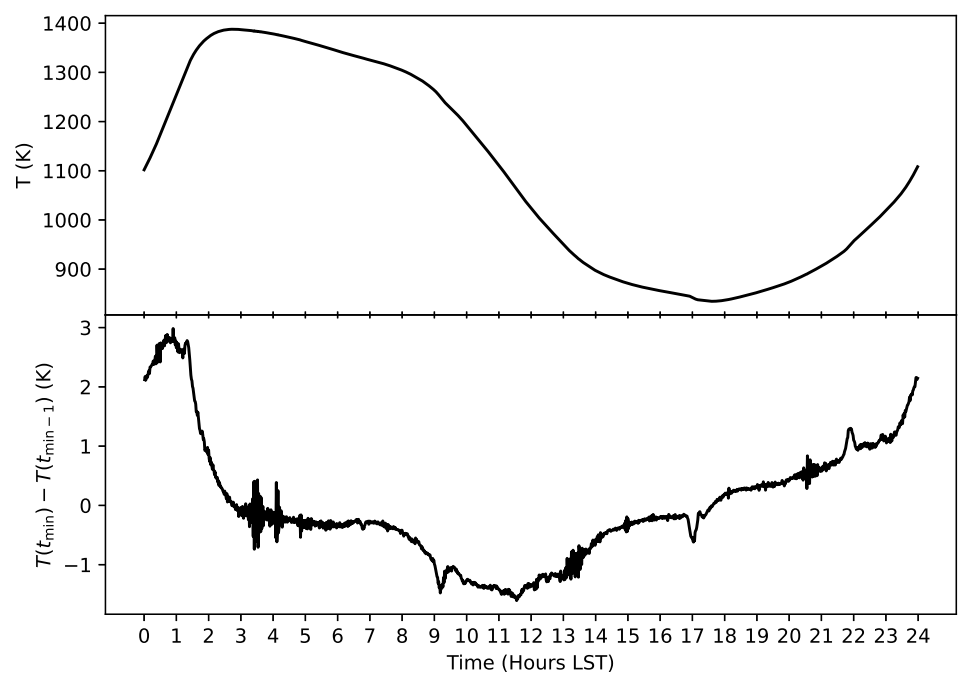

Fig. 3: Diurnal variation (top panel) and difference (bottom panel) of the Galactic diffuse emission in 1 minute interval over 24 hours at $100 \mathrm{MHz}$, predicted by the simulations of de Oliveira-Costa et al. (2008). The 21CMA site and an imaginary observation on January 1, 2021 are taken for this illustration.

Here we explore further the possibility because our method is entirely unaffected by beam pattern. The new concern is, however, whether the variation of the Galactic noise is actually visible/observable during the latter two position switching at any time of a day.

Figure 3 shows an example of the diurnal variation of the Milky Way in 1 minute interval over 24 hours at $100 \mathrm{MHz}$, in which an imaginary observation is made on January 1, 2021 at the location of the 21CMA site (longitude 68.68 , latitude 42.93 ${ }^{\circ}$ ) (Huang et al. 2016, Zheng et al. 2016) and a Gaussian beam of FWHM $=43.3^{\circ}$ is assumed for the receiving antenna. Our demonstration is based on the diffuse Galactic radio emission model proposed by de Oliveira-Costa et al. (2008). It appears that the amplitude of the sky-averaged brightness temperature of the Milky Way can reach $\sim 1000 \mathrm{~K}$ even within 1 minute, suggesting that the diurnal motion of the Galactic signal could be used as an ideal calibrator in terms of magnitude alone for the EoR experiment.

Denoting the sky temperature $\bar{T}_{\text {sky }}$ as the foreground component $\bar{T}_{f}$ plus the EoR signal $T_{21 \mathrm{~cm}}$ and keeping the ratio of $T_{21 \mathrm{~cm}} / \bar{T}_{f}$ to the first order, we can rewrite Eq.(17) as

$$
\begin{aligned}
\frac{T_{3}-T_{2}}{T_{3}-T_{\text {sys }}}= & \frac{\Delta \bar{T}}{\bar{T}_{f}}\left\{1-\frac{1}{2} \frac{\Delta \bar{T}}{\bar{T}_{f}}+\frac{1}{4}\left(\frac{\Delta \bar{T}}{\bar{T}_{f}}\right)^{2}+O\left(\frac{\Delta \bar{T}}{\bar{T}_{f}}\right)^{3}\right. \\
& \left.-\frac{T_{21 c m}}{\bar{T}_{f}}\left[1-\frac{\Delta \bar{T}}{\bar{T}_{f}}+\frac{3}{4}\left(\frac{\Delta \bar{T}}{\bar{T}_{f}}\right)^{2}+O\left(\frac{\Delta \bar{T}}{\bar{T}_{f}}\right)^{3}\right]\right\} .
\end{aligned}
$$

In a logarithmic expression, the above equation takes the form of

$$
\ln \frac{T_{3}-T_{2}}{T_{3}-T_{\text {sys }}}=\ln \left(\frac{\Delta \bar{T}}{\bar{T}_{f}}\right)+\mathcal{F}\left(\frac{\Delta \bar{T}}{\bar{T}_{f}}\right)-\frac{T_{21 c m}}{\bar{T}_{f}},
$$

where $\mathcal{F}$ is an analytical function of $\Delta \bar{T} / \bar{T}_{f}$. Subtracting a best-fitted smooth component or a polynomial in frequency domain would allow us to find the EoR signal $T_{21 \mathrm{~cm}}$ calibrated by the sky averaged foreground brightness temperature $\bar{T}_{f}$ (rather than the variation $\Delta \bar{T}$ ). Yet, application of this method depends critically on an accurate understanding and modelling of the diurnal motion of the Galactic 
diffuse radiation in the frequency range of 50-200 MHz. The typical accuracy in current models is between 5\% and 15\% across the entire sky (de Oliveira-Costa et al.2008, Zheng et al.2017). The error in the global EoR signal resulting from this inaccuracy can be estimated from Eq.(19)

$$
\frac{d T_{21 \mathrm{~cm}}}{T_{21 \mathrm{~cm}}}=\frac{d \bar{T}_{f}}{\bar{T}_{f}}+O(1) \frac{d \Delta \bar{T}_{f}}{\Delta \bar{T}_{f}} \approx 2 \frac{d \bar{T}_{f}}{\bar{T}_{f}},
$$

in which the diurnal motion term shares the same accuracy as the Galactic emission one. Therefore, the error in $T_{21 \mathrm{~cm}}$ from current modelling of the Galactic diffuse radiation is less than $30 \%$.

In order to examine whether this algorithm allows us to efficiently extract the EoR signal, we simulate a set of measurements of up to 72 hours with the observational parameters in Fig.3 and the setup in Fig.2. For the $21 \mathrm{~cm}$ global background, we adopt a phenomenal model of Gaussian absorption profile (Bowman et al.2018)

$$
T_{21 c m}=a \exp \left[-\frac{\left(\nu-\nu_{21}\right)^{2}}{2 \sigma^{2}}\right],
$$

where $a=-150 \mathrm{mK}, \nu_{21}=78.3 \mathrm{MHz}$ and $\sigma=5$ are the amplitude, peak position and deviation of the absorption trough, respectively. We also run a flattened Gaussian profile for comparison:

$$
T_{21 c m}=a\left\{\frac{1-\exp [-\tau \exp (b)]}{1-\exp (-\tau)}\right\}
$$

in which

$$
b=\frac{4\left(\nu-\nu_{0}\right)^{2}}{w^{2}} \log \left[-\frac{1}{\tau} \log \left(\frac{1+\exp (-\tau)}{2}\right)\right] .
$$

All the parameters $a, \nu_{0}, w$ and $\tau$ can be fixed by fitting the observed absorption trough from EDGES 2018: $a=-520 \mathrm{mK}, \nu_{0}=78.3 \mathrm{MHz}, w=20.7 \mathrm{MHz}$, and $\tau=7$.

In the mock observation, three-position switch is performed in a time interval of 1 minute each with bandwidth of $50 \mathrm{kHz}$, namely, a total of 3000 sampling points over $150 \mathrm{MHz}(50-200 \mathrm{MHz})$ bandpass are taken. We assume a constant receiving system temperature of $T_{\text {sys }}=50 \mathrm{~K}$. A series of 'observing' data $\left(T_{1}^{i}=T_{\mathrm{sys}}^{i}=50 \mathrm{~K}, T_{2}^{i}, T_{3}^{i} ; i=1,2, \ldots, 3000\right)$ would be collected every three minutes. For each set of measurement ( 3 minutes) we find the best-fit polynomial in frequency domain from $\ln \left[\left(T_{3}^{i}-T_{2}^{i}\right) /\left(T_{3}^{i}-T_{\text {sys }}^{i}\right)\right]$ and then obtain the residual $\Delta^{j}$. We accumulate and average the residuals over a sufficiently long time to reach the desired sensitivity. Eventually, the $21 \mathrm{~cm}$ global signals can be extracted through $\bar{T}_{21 \mathrm{~cm}}=-\bar{T}_{f} \bar{\Delta}$. Fig. 4 shows the low-frequency sky model, $T_{\mathrm{sky}}=T_{\mathrm{sys}}+\bar{T}_{21 \mathrm{~cm}}+\bar{T}_{f}$, two input models of the cosmic EoR background $\bar{T}_{21 \mathrm{~cm}}$ described by Eqs.(21) and (22), respectively, and the recovered signals, in which mock observations over 72 hours or 1440 sets of measurements are simulated.

Technically, we first employ the Savitzky-Golay filter with a window of $\sim 10 \mathrm{MHz}$ in the fitting of $\ln \left[\left(T_{3}^{i}-T_{2}^{i}\right) /\left(T_{3}^{i}-T_{\text {sys }}^{i}\right)\right]$ over 50-200 MHz. It turns out that the residual errors are within an acceptable level except in a certain frequency range where the radiation is mixed with the background EoR signal, reflected by some relatively large dispersions and oscillations. We then perform the second polynomial fitting by blanking the data in the 'noise' region. The residual between the measurement and the newly fitted polynomial will be accumulated to extract the EoR signal.

Finally, one may argue that the Galactic diffuse emission and its diurnal motion demonstrate complex spatial structures and time variation, while the $21 \mathrm{~cm}$ radiation from EoR is isotropic in the sky and remains almost time-invariant. Apparently, the external calibrator no longer mimics the background radiation. For this reason, if the measurements are made during the Galaxy down, the effect of the Galactic structures may be largely reduced. On the other hand, if the spatial response of the receiving antenna, $A(\theta, \phi, \nu)$, is designed to be a smooth function of observing frequency, the above algorithm can still be used to extract the EoR signal, as demonstrated by our mock observation. 


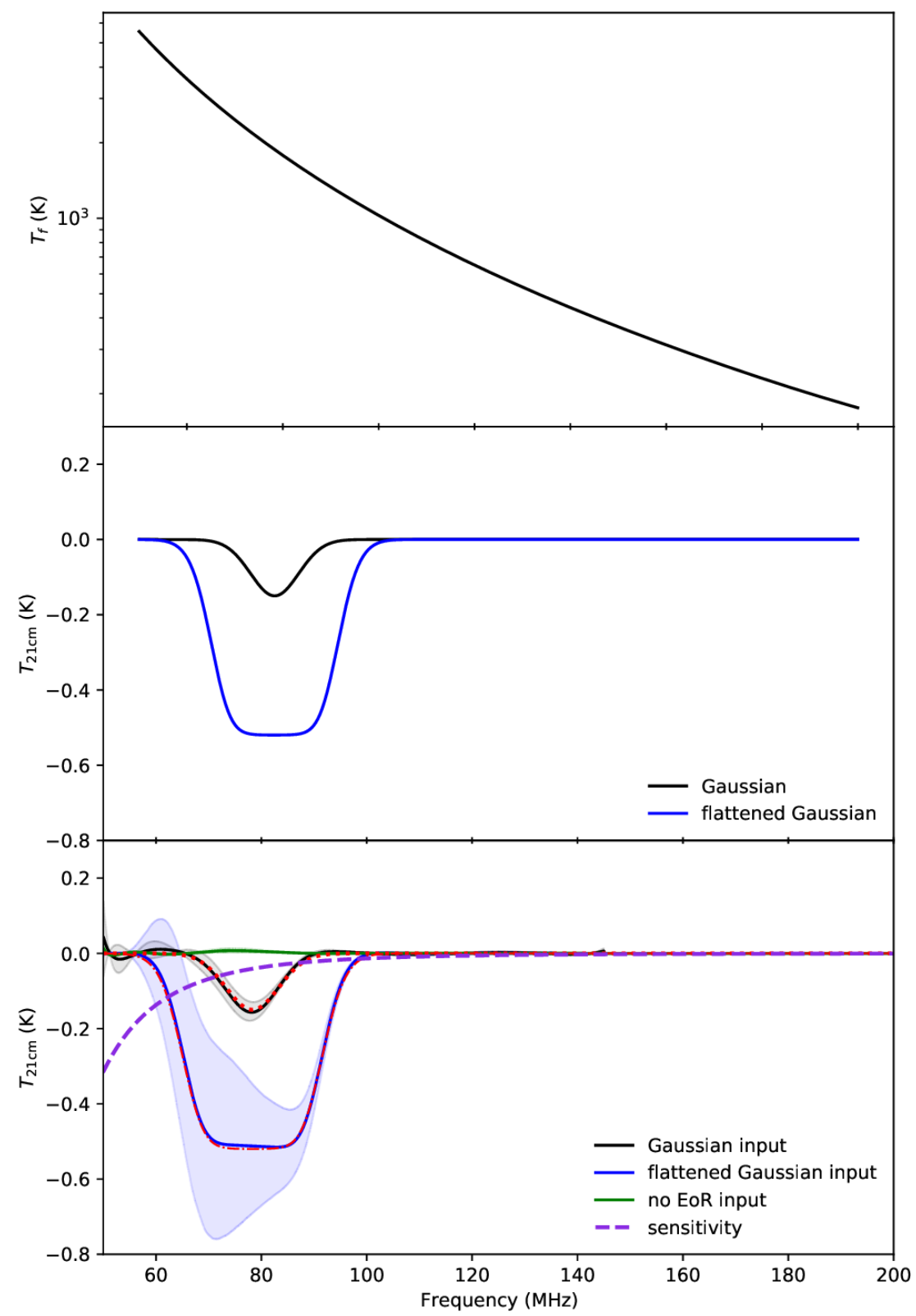

Fig. 4: Top panel: The input Galactic diffuse emission model (2008) as a function of observing frequency. Middle panel: The input $21 \mathrm{~cm}$ EoR signal described by a Gaussian absorption profile (black lines) and a flattened Gaussian absorption profile (blue line), respectively. Bottom panel: The recovered $21 \mathrm{~cm}$ global signals from 72 hours (1440 sets of measurements) of mock observations. The shadow regions in each case indicate the dispersions represented by 14-86 percentile of the recovered signal around the average (solid line) over 1440 measurements. Overlaid dotted/dot-dashed lines are the corresponding original input signals. The large dispersions arise partially from the 'calibrator' $T_{f}$, which demonstrates a diurnal variation as shown in Fig.3. For comparison, we have also shown the result for the Galactic emission alone as the input signal using the same algorithm of signal extraction. Dashed line indicates the predicted sensitivity based on a bandwidth of $\Delta \nu=1 \mathrm{MHz}$ for an integration time of 24 hours, in which an efficiency of $1 / 3$ has been used. 


\section{OUTDOOR CALIBRATION: POLARIZATION}

Measurement of polarization plays a key role in modeling and subtracting foreground contamination in EoR experiment. It has been realized that the low-frequency foregrounds are dominated by synchrotron radiation and thus polarized because of the existence of magnetic field, while the cosmic CD/EoR signal is thermal emission/absorption and therefore unpolarized. A naive approach that is yet worthy of further exploration is to use this unique feature as a calibrator in EoR experiment. The same three-position switch measurements can be once again employed except that latter two steps are switched between two polarized states. There are two ways to achieve this goal through either (1) a pair of perpendicularly spaced and linearly polarized dipoles or (2) a linearly polarized dipole mounted on a 90-degree rotating platform. The whole system is placed in the underground shallow anechoic chamber, similar to the experimental environment discussed above.

The first design requires two perpendicularly spaced and linearly polarized dipoles and hence two backend receivers but the measurements can be made simultaneously. One can also carry out the measurement in two separate steps by sharing the same receiver to reduce the systematics. Yet, for the latter the measurement would also contain a time-varying component from the diurnal motion of the Galactic emission that we have discussed in the above section. The calibrator $\Delta \bar{T}$ in Eq.(19) is eventually a combination of both the polarized and diurnal variations of the sky. For the former, while a simultaneous measurement of two orthogonal polarization components is unaffected by the diurnal motion of the Galactic emission, the whole experiment actually involves two independent channels (dipoles, receivers and DAQ). Systematics could be introduced even if they are fabricated to be identical. Another concern for both designs arises from the possible cross-talk between two perpendicularly spaced dipoles.

The second design is based on a single dipole mounted on a 90-degree rotating platform. Threeposition switch operations are also applied, and we only need to replace the second and third steps by measuring two orthogonal X-Y polarized components $T_{X}$ and $T_{Y}$ at time $t=t_{2}$ and $t=t_{3}$, respectively, which is achieved by rotating the single-polarized linear dipole by 90 degrees. Although the receiver can be assumed to be stable and unchanged during the rotation, the variation of the sky brightness temperature in such a measurement, however, includes both the polarized sky $\Delta \bar{T}_{P}=T_{Y}-$ $T_{X}$ and diurnal motion of the Galactic emission $\Delta \bar{T}_{\mathrm{MK}}$. Replacing $\Delta \bar{T}$ by $\Delta \bar{T}=\Delta \bar{T}_{P}+\Delta \bar{T}_{\mathrm{MK}}$, Eq.(19) should still remain valid and can be used to obtain the $21 \mathrm{~cm}$ EoR signal, provided that the polarization signal does not break down the smooth spectrum assumption.

Unfortunately, the foreground polarization may result in a frequency-dependent spectrum that further exhibits a rapid and irregular variation as a function of Faraday depth when the linearly polarized radiation travels through the Galactic magnetic field (Rybicki \& Lightman 1986, Carucci et al. 2020). The exact magnitude of this polarization leakage into the EoR signal is still uncertain (e.g. Moore et al. 2013, 2017; Asad et al. 2015). Recent simulation involving a more realistic model of Galactic polarized synchrotron emission and a single polarization antenna suggested that the polarized foreground not only can give rise to a complex frequency structure but also produce an enhanced and distorted 21 $\mathrm{cm}$ absorption though similar to the anomalous profile detected by the EDGES experiment (Spinelli et al. 2018). Before further investigations are needed to clarify the issue, for the time being we would not recommend the foreground polarization as an external calibrator in the global EoR experiment.

\section{DISCUSSION AND CONCLUSIONS}

We have presented a conceptual design study of four types of external calibrators for the $21 \mathrm{~cm}$ EoR experiment aiming at measuring the globally averaged sky brightness in frequency range of 50-200 MHz. Unlike the internal calibrators widely adopted in current EoR experiments, external calibrator seeks to mimic a radiation field similar to that of the EoR signal. This allows one to completely remove instrumental effect such as direction- and frequency-dependent beam of the antenna, frequency-dependent gain of the receiver, and even the unstability of the system if measurements are implemented within a short time interval. The whole system is placed in an underground shallow anechoic chamber with an open/close ceiling to further reduce the environmental effect such as RFI and ground radiation/reflection. 
Conventional three-position switch measurements are implemented among system noise, external calibrator and low-frequency sky.

We have explored two external calibrators for each of the indoor and outdoor calibration. It appears that two types of calibrator in the indoor calibration based on artificial emitting sources fail to meet our purpose, due to either small sky coverage or difficulties of engineering realization. Outdoor calibration relies on celestial astronomical sources in low frequencies. The polarized foreground especially the Milky Way as a calibrator is not recommended because the Faraday rotation of the Galactic magnetic would break down the smooth spectrum scenario, a key to remove the foreground contamination, though it is still uncertain whether the magnitude of such a polarization leakage is comparable to that of the EoR signal. Therefore, the possible candidate in the outdoor calibration turns to be the diurnal motion of the Galactic diffuse emission. Indeed, the Galactic radiation in low frequency shares the same sky coverage as that of the EoR signal. This provides a possibility of eliminating the beam effect of the receiving antenna, an advantage over the internal calibrator in current EoR experiments. While the application of this method depends on our understanding and modelling of the diurnal motion of the Galactic diffuse radiation in the frequency range of 50-200 MHz, the extraction of the EoR signal is actually unaffected by the exact magnitude variation $\Delta \bar{T}$ in radiation due to diurnal motion of the Galaxy, as shown in Eq.(19), provided that $\Delta \bar{T}$ does not introduce any complex spectral structures. Indeed, subtraction of the best-fitted foreground, say, a polynomial, from Eq.(19) will unveil the EoR signature calibrated by the total intensity of the foreground. Yet, regardless of its same sky coverage as that of the EoR, the Galaxy demonstrates complex spatial structures. To what extent the Galactic spatial structures affect the measurement of the EoR signal needs further investigation.

Now it is technically feasible to construct and conduct a new experiment towards the detection of the global $21 \mathrm{~cm}$ EoR signal using the Galactic emission and its diurnal motion as an external calibrator. The antenna, receiver and data acquisition system have been well developed by the existing experiments such as BIGHORNS, EDGES, LEDA, $\mathrm{PRI}^{z} \mathrm{M}$, SARAS and SCI-HI. The most expensive component in this experiment is perhaps the underground shallow anechoic chamber - the platform to host the whole system. It would be better that the measurements are implemented in the 'Galaxy down' phase to maximally reduce the possible influence of the Galactic spatial structures. We have already raised sufficient funds to support such an experiment, and it is hoped that the design of the system can be completed recently and the experiment can start to collect data in about two years.

Acknowledgements This work is supported by the Key Projects of Frontier Science of Chinese Academy of Sciences under grant No. QYZDY-SSW-SLH022, the Strategic Priority Research Program of Chinese Academy of Sciences under grant No. XDB23000000, and the National Key R\&D Program of China under grant No. 2018YFA0404601.

\section{References}

Asad, K. M. B., Koopmans, L. V. E., Jelic, V., et al. 2015, MNRAS, 451,3709

Barkana, R., 2018, Nature, 555, 71

Bernardi, G., Zwart, J. T. L., Price, D., et al. 2016, MNRAS, 461, 2847

Bowman, J. D., Rogers, A. E. E., \& Hewitt, J. N., 2008, ApJ, 676,1

Bowman, J. D., \& Rogers, A. E. E., 2010, Nature, 468, 796

Bowman, J. D., Rogers, A. E. E., Monsalve, R. A., Mozdzen, T. J., Mahesh, N., 2018, Nature, 555, 67

Bradley, R. F., Tauscher, K., Rapetti, D., \& Burns, J. O., 2019, ApJ, 874, 153

Carucci, I. P., Irfan, M. O., \& Bobin, J., 2020, MNRAS, 499, 304

Chang, C., Monstein, C., Refregier, A., et al. 2015, PASP, 127, 1131

Chapman, E., Abdalla, F. B., Harker, G., et al. 2012, MNRAS, 423, 2518

de Oliveira-Costa, A., Tegmark, M., Gaensler B., et al. 2008, MNRAS, 388, 247

Ewall-Wice, A., Chang, T. -C., Lazio, J., et al. 2018, ApJ, 868, 63 
Feng, C., \& Holder, G., 2018, ApJL, 858, L17

Fialkov, A., Barkana, R., \& Cohen, A., 2018, Physical Review Letters, 121, 011101

Fialkov, A., \& Barkana, R., 2019, MNRAS, 486, 1763

Garza, S., Trung, V., Ewall-Wice, A. M., et al. 2018, American Astronomical Society, AAS Meeting \#231, id. 153.13

Harker, G. J. A., Pritchard, J. R., Burns, J. O., \& Bowman, J. D., 2012, MNRAS, 419, 1070

Huang, Y., Wu, X. -P., Zheng, Q., Gu, J. -H., Xu, H., 2016, Research in Astron. Astrophys. (RAA), 16, 36

Jacobs, D. C., Burba, J., Bowman, J., et al. 2016, arXiv:1610.02607

Mckeinley, B., Bernardi, G., Trott, C. M., et al. 2018, MNRAS, 481, 5034

Moore, D. F., Aguirre, J. E., Parsons, A. R., Jacobs, D. C., \& Pobers, J. C., 2013, ApJ, 769, 154

Moore, D. F., Aguirre, J. E., Kohn, S. A., et al., 2017, ApJ, 836, 154

Patra, N., Subrahmanyan, R., Raghunathan, A., \& Udaya Shankar, N., 2013, Experimental Astronomy, 36, 319

Philip, L., Abdurashidova, Z., Chiang, H. C., et al. 2019, Journal of Astronomical Instrumentation, 8, 1950004

Presley, M. E., Liu, A., \& Parsons, A. R., 2015, ApJ, 809, 18

Price, D. C., Greenhill, L. J., Fialkov, A., et al. 2018, MNRAS, 478, 4193

Pritchard, J. R., \& Loeb, A., 2010, Physical Review D, 82, 023006

Rogers, A. E. E., \& Bowman, J. D., 2012, Radio Science, 47, RS0K06

Rybicki, G. B., \& Lightman, A. P., 1979, Radiative Processes in Astrophysics (Wiley, New York)

Singh, S., Subrahmanyan, R., Udaya Shankar, N., \& Raghunathan, A., 2015, ApJ, 815, 88

Shaver, P. A., Windhorst, R. A., Madau, P., \& de Bruyn, A. G., 1999, MNRAS, 345, 380

Singh, S., Subrahmanyan, R., Udaya Shankar, N., et al. 2018, Experimental Astronomy, 45, 269

Spinelli, M., Bernardi, G., \& Santos, M. G., 2018, MNRAS, 479, 275

Sokolowski, M., Tremblay, S. E., Wayth, R. B., et al. 2015, Publications of the Astronomical Society of Australia, 32, e004

Virone, G., Lingua, A. M., Piras, M., et al. 2014, IEEE Antennas and Wireless Propagation Letters, 13, 169

Voytek, T. C., Natarajan, A., Jauregui Garcia, J. M., Peterson, J. B., \& Lopez-Cruz, O., 2014, ApJL, 782, L9

Wang, X., Tegmark, M., Santos, M. G., \& Knox, L., 2006, ApJ, 650, 529

Zheng, H., Tegmark, M., Dillon, J. S., et al. 2017, MNRAS, 464, 3486

Zheng, Q., Wu, X. -P., Johnston-Hollitt, M., Gu, J. -H., Xu, H., 2016, ApJ, 832, 190

This paper was prepared with the RAA LATEX macro v1.2. 